\title{
Obtenção da derivada direcional radial e sua aplicação na atenuação do ground-roll
}

Rafael M.Paulo,, CPGG/UFBA, Milton J. Porsani, CPGG/UFBA

Copyright 2019, SBGf - Sociedade Brasileira de Geofísica

This paper was prepared for presentation at the $16^{\text {th }}$ International Congress of the Brazilian Geophysical Society, held in Rio de Janeiro, Brazil, 19-22 August 2019.

Contents of this paper were reviewed by the Technical Committee of the $16^{\text {th }}$ International Congress of The Brazilian Geophysical Society and do not necessarily represent any position of the SBGf, its officers or members. Electronic reproduction or storage of any part of this paper for commercial purposes without the written consent of The Brazilian Geophysical Society is prohibited.

\section{Abstract}

The present work shows an attenuation method for ground-roll, the radial directional derivative, calculated from the interpolation Shepard's method. This noise has high amplitudes and prevents good visualization of the reflections. The filter uses linear coherence of noise, as well as its property of having low frequencies, in order to be applied. The radial directional derivative method has some advantages in the attenuation of the ground-roll, when compared to the results obtained by low cut and FK filtering methods. The first advantage is that the radial directional derivative filter does not require changes of domain, and thus does not present noises related to these changes. The second advantage is that despite the ground-roll be attenuated, the low frequency information are not totally lost and can be used in the interpretation of the data, the method does not use positions perturbations for the calculation of the derivative and using an analytical expression of the directional derivative of the Shepard method's weight function.

\section{Introdução}

O ground-roll é um ruído do dado sísmico causado pela onda Rayleigh, este ruído mascara informações de interesse do dado e possui certas características que podem ser exploradas para sua filtragem. Estas características são:

- Altas amplitudes.

- Baixas velocidades quando comparadas com a onda P.

- Baixas frequências.

- Coerência linear em uma seção de tiro comum.

O filtro da derivada direcional radial utiliza as propriedades de baixas frequências e da coerência linear do ruído. $O$ filtro consiste em uma janela móvel que percorre o dado, com números impares de linhas e colunas e iguala seu ponto central à soma das derivadas direcionais dos pontos contidos na janela. A direção da derivada é escolhida a partir do vetor unitário que parte do centro da janela até um foco. Este foco será igual independente da posição da janela, segundo (Santos, 2014). O método da derivada direcional possui um melhor resultado se o foco for amarrado à origem do ruído. A Figura 1 ilustra a estrutura do método. A reta que passa entre o centro da janela e $P_{f}$ mostra a direção da derivada direcional.

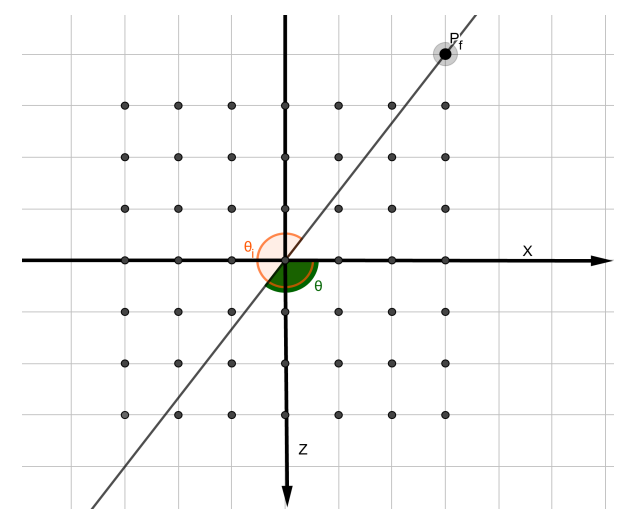

Figura 1: Exemplificação da estrutura do filtro

O filtro, como ilustrado, atua de forma radial e por ser um método de derivada, atenua mais as baixas frequências e devido a estas características este filtro atua sobre o ground-roll.

Para o teste do método a derivada direcional foi aplicada no trinta e nove da linha L5090 da bacia do Tacutu.

O método da derivada direcional 2D foi utilizado por (Manenti, 2013), que utilizou a transformada radial, introduzido por (Ottolini, 1979) para o processo de migração, houve, desta forma, um remapeamento de amplitudes utilizando o método de interpolação de Shepard no sismograma, reorganizando, assim, os eventos lineares em eventos verticais de baixa frequência e posteriormente derivando o dado utilizando a interpolação de Shepard para calcular operadores a frente e atrás do ponto a ser derivado, este método depende de uma pertubação $\delta$ que representa a distância entre os pontos preditos e o ponto central. O método também foi utilizado por (Santos, 2014) através de outra abordagem, sem a necessidade de mudança de domínios, para tanto, foi escolhido um foco no sismograma e o dado foi derivado direcionalmente com relação a este foco, para tanto foi calculada as derivadas do dado em $x$ e $t$ utilizando o método de Shepard para calcular as amplitudes de pontos que distam $\delta$ do ponto a ser derivado, com a derivada sendo a diferença do ponto a frente e atrás do ponto central, e assim multiplicando estas derivadas pelos respectivos componentes do vetor unitário. Ambos os trabalhos utilizam uma estrutura de janela móvel que passeia pelo dado realizando estas derivadas.

O presente trabalho realiza a derivada direcional 2D sem a 
necessidade de mudanças de domínio, desta forma não havendo ruídos numéricos devido a remapeamentos de amplitude desta natureza e sem utilizar pertubações de distância para o cálculo da derivada, sem utilizar o $\delta$, para tanto foi deduzida uma expressão analítica da derivada direcional da função peso da interpolação de Shepard.

\section{Teoria}

O método de interpolação baseado no inverso da distância, assume que pontos próximos influenciam mais na interpolação quando comparados a pontos distantes. Um dos métodos mais simples de interpolação que utiliza este conceito é chamado de método de Shepard.

Dado um grid de $\mathrm{N}$ pontos espaçados de forma não necessariamente regular contendo pontos dispostos no $\mathbb{R}^{2}$, distribuídos ao longo do plano $x-z$ com cada um destes pontos possuindo uma amplitude conhecida. Ou seja, o j-ésimo ponto terá uma amplitude $A_{j}$, desta forma para os espaços entre os pontos do grid, onde não se tem informações da amplitude, esta pode ser obtida através do método de interpolação de Shepard. Assim dado um ponto do espaço localizado em $P(x, z)$, segundo (Shepard, 1968) a amplitude associada ao ponto será dada pela equação (1):

$$
A(x, z)=\sum_{j=1}^{N} A_{j} w_{j}
$$

Onde $w_{j}$ é a função peso associada a cada ponto do grid. E é dada pela equação (2):

$$
w_{j}=\frac{d_{j}^{-p}}{\sum_{i=1}^{N} d_{i}^{-p}}
$$

Neste trabalho $d_{i}$ representa a distância entre o ponto $P$ e o i-ésimo é dado por (3)

$$
d_{i}=\sqrt{\left(x_{i}-x\right)^{2}+\left(z_{i}-z\right)^{2}}
$$

Pela definição da função $A(x, z)$, dado pela equação (1), é possível notar que qualquer derivada parcial da mesma depende apenas do somatório das derivadas parciais de $w_{j}$, assim:

$$
\frac{\partial A(x, z)}{\partial x}=\sum_{j=1}^{N} A_{j} \frac{\partial w_{j}}{\partial x}
$$

Trocando $x$ por $z$ na equação (4) é obtida a derivada parcial em $z$.

As derivadas parciais da função peso devido a uma distribuição bidimensional de pontos são dadas pelas equações (5) e (6).

$$
\begin{aligned}
& \frac{\partial w_{j}}{\partial x}=p\left[\frac{w_{j}\left(x_{j}-x\right)}{d_{j}^{2}}-w_{j}^{2} d_{j}^{p} \sum_{i=1}^{N} \frac{x_{i}-x}{d_{i}^{p+2}}\right] \\
& \frac{\partial w_{j}}{\partial z}=p\left[\frac{w_{j}\left(z_{j}-z\right)}{d_{j}^{2}}-w_{j}^{2} d_{j}^{p} \sum_{i=1}^{N} \frac{z_{i}-z}{d_{i}^{p+2}}\right]
\end{aligned}
$$

O sismograma contém dados igualmente espaçados, possuindo assim propriedades que podem ser utilizadas para calcular de forma mais fácil a derivada direcional do dado, considerando uma janela atuando em um sismograma contendo $2 L_{x}+1$ amostras, numeradas de $-L_{x}$ até $L_{x}$, regularmente espaçadas de $\Delta x$ em $x$ e com $2 L_{z}+1$ amostras, numeradas de $-L_{z}$ até $L_{z}$, regularmente espaçadas de $\Delta z$ em $z$. Considerando que existem amostras de amplitude em toda a janela, exceto no centro da janela, denotado $P_{0}$, e este ponto é onde se deseja fazer a interpolação.

Desta forma é conveniente modificar a notação de $w_{j}$ e $d_{j}$ para:

$$
\begin{gathered}
w_{j} \rightarrow w_{j k} \\
d_{i} \rightarrow d_{i l} \\
\sum_{i=1}^{N} \frac{1}{d_{i}} \rightarrow \sum_{i=-L z l=-L x}^{L z} \sum^{L x} \frac{1}{d_{i l}}
\end{gathered}
$$

$d_{i l}$ é escrito segundo a equação (7):

$$
d_{i l}=\sqrt{(l \Delta x)^{2}+(i \Delta z)^{2}}
$$

$w_{j k}$ é escrito segundo a equação (8):

$$
w_{j k}=\frac{d_{j k}^{-p}}{\sum_{i=-L z}^{L z} \sum_{l=-L x}^{L x} \frac{1}{d_{i l}}}
$$

As derivadas parciais da função peso, avaliadas em $P_{0}$ são dadas por:

$$
\begin{array}{r}
\frac{\partial w_{j k}}{\partial x}=p\left[\frac{w_{j k} k \Delta x}{d_{j k}^{2}}-w_{j k}^{2} d_{j k}^{p} \sum_{i=-L z l=-L x}^{L z} \sum_{i l}^{L x} \frac{l \Delta x}{d_{i l}^{p+2}}\right] \\
\frac{\partial w_{j k}}{\partial z}=p\left[\frac{w_{j k} j \Delta z}{d_{j k}^{2}}-w_{j k}^{2} d_{j k}^{p} \sum_{i=-L z}^{L z} \sum_{l=-L x}^{L x} \frac{i \Delta z}{d_{i l}^{p+2}}\right]
\end{array}
$$

Os índices $i$ e $l$ dos somatórios nas equações (9) e (10), não são simultaneamente iguais a zero. Pois trabalhase como se não houvesse informações da amplitude no ponto central da janela. Devido a simetria da janela os somatórios destas equações irão se anular, desta forma o gradiente da função peso para este tipo de dado é mostrado na equação (11):

$$
\vec{\nabla} w_{j k}=p \frac{w_{j k}}{d_{j k}^{2}}(k \Delta x, j \Delta z)
$$

A direção que parte do centro da janela até á fonte do ground-roll será dada a partir do vetor unitário $\vec{u}$. Dado pela equação (12):

$$
\vec{u}=\frac{1}{\sqrt{\left(x_{f}-x_{0}\right)^{2}+\left(z_{f}-z_{0}\right)^{2}}}\left(x_{f}-x_{0}, z_{f}-z_{0}\right)
$$

Desta forma a derivada direcional, neste trabalho denotada de $D_{u}$, será dado pela equação (13):

$$
D_{u} w_{j k}={ }^{1} w_{j k}=\frac{p w_{j k}\left(k \Delta x\left(x_{f}-x_{0}\right)+j \Delta z\left(z_{f}-z_{0}\right)\right)}{d_{j k}^{2} \sqrt{\left(x_{f}-x_{0}\right)^{2}+\left(z_{f}-z_{0}\right)^{2}}}
$$

A partir das equações (4) e (13) é possível obter a derivada direcional do ponto interpolado, como mostrado na equação (14):

$$
D_{u} A\left(x_{0}, z_{0}\right)=\sum_{j=-L z / 2}^{L z / 2} \sum_{k=-L x / 2}^{L x / 2} A_{j k} 1^{1} w_{j k}\left(x_{0}, z_{0}\right)
$$


Assim, é possível notar que na equação (14) o cálculo da derivada direcional radial tem um fator de amplitude dado por $A_{j k}$ e um fator geométrico dado por ${ }^{1} w_{j k}$

Em um sismograma é possível atribuir um diferente ${ }^{1} w_{j k}$ para cada localização do centro da janela, desta forma, considerando que um tiro possui $N_{x}$ traços e $N_{z}$ amostras verticais, e considerando que um ponto $P_{l}$ do sismograma se localiza em uma linha $m$ e uma coluna $n$ este ponto terá uma numeração $l=m+(n-1) N_{z}$, quando o centro da janela passar por $P_{l}$ o operador ${ }^{1} w_{j k l}$ de um ponto $(j, k)$ da janela do operador será dado pela equação (15):

$$
{ }^{1} w_{j k l}=\frac{p w_{j k}\left(k \Delta x\left(x_{f}-x_{l}\right)+j \Delta z\left(z_{f}-z_{l}\right)\right)}{d_{j k}^{2} \sqrt{\left(x_{f}-x_{l}\right)^{2}+\left(z_{f}-z_{l}\right)^{2}}}
$$

Assim considerando, uma matriz tridimensional $\mathbf{W}_{\mathbf{1}}$ com dimensões $\left(2 L_{z}+1,2 L_{x}+1, N_{x} N_{z}\right)$, cada ponto da matriz será dado por $\mathbf{W}_{\mathbf{1}}(j, k, l)={ }^{1} w_{j k l}, \mathbf{W}_{\mathbf{1}}$ será uma matriz onde cada elemento corresponde ao fator geométrico da equação (14), quando o centro da janela está localizada no ponto $P_{l}$. Assim, como esta matriz só depende de fatores geométricos do sismograma, considerando uma linhas de tiros com números de traços e amostras por traços constantes em cada tiro, então é necessário se calcular $\mathbf{W}_{\mathbf{1}}$ para um tiro, e esta matriz será igual para os demais tiros. Outra otimização pode ser feita nos cálculos de $d_{i l}$ e $w_{j k}$, pelas equações (7) e (8), $d_{i l}$ e $w_{j k}$ dependem apenas de fatores internos da janela, desta forma podem ser calculados uma vez e serão válidas para qualquer posição da janela em um tiro, e para qualquer tiro na linha.

O método da derivada direcional possui grande dependência com relação ao operador vetor unitário, este, possui amplitude constante no entanto não apresenta fase constante.

\section{Resultados}

Para testar o método foi aplicado a derivada direcional na linha L5090 da bacia do Tacutu, este dado possui uma forte influência de ground-roll, sendo um bom teste para o filtro.

Como o terreno onde foi realizada a aquisição é plano, não foi necessária a realização da correção estática para garantir a linearidade do ground-roll. A filtragem foi realizada após a correção de amplitude, a correção utilizada neste trabalho foi a correção AGC. O arranjo da aquisição é do tipo split-spread simétrico, desta forma o $x_{f}$ foi escolhido como sendo igual a 48 , que corresponde a metade do numero de traços, enquanto $0 z_{f}$ ocorre por volta da amostra 10, o dado possui 96 traços e 1001 amostras por traço. A Figura 2 mostra a comparação do tiro 39 desta linha, antes e após a aplicação da derivada direcional. É possível notar que a Figura 2a possui um maior conteúdo de eventos lineares de baixa frequência e alta amplitude que foram atenuadas na Figura 2b, desta forma as reflexões puderam ser evidenciadas nesta Figura, a exemplo de algumas reflexões rasas, que ocorrem por volta de 0,5 segundos e uma reflexão que ocorre por volta de 1,5 segundos são exemplos, as reflexões que mais sofrem com a contaminação do ground-roll são as mais profundas, assim, as reflexões que foram mais evidenciadas são as que ocorrem abaixo de 2,5 segundos. É possível notar a ocorrência da perda de amplitude da seção na Figura $2 b$ quando comparada a seção da Figura 2a, isto é uma característica do método da derivada, no entanto, não constitui um grande problema ao processamento sísmico, pois isto ocorre no tiro como um todo, podendo ser facilmente corrigido em futuras etapas do processamento.

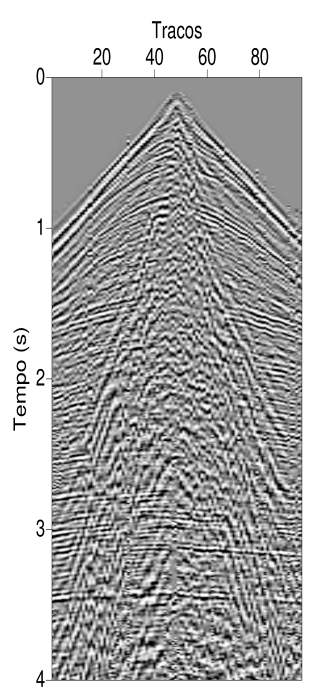

(a)

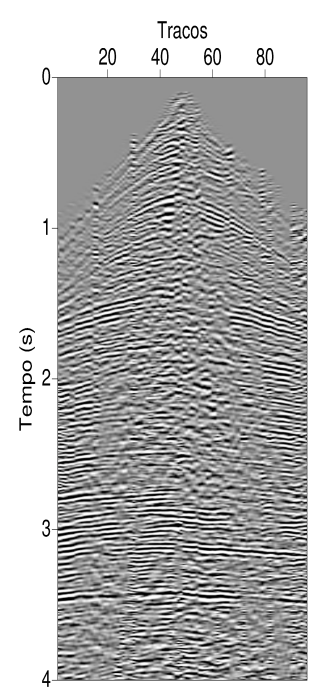

(b)
Figura 2: (a) tiro antes da aplicação da derivada (b) tiro após a aplicação do filtro.

A Figura 3 mostra o espectro de potência da linha antes (em preto) e após(em azul) a filtragem, é possível notar mais uma vez a perda de energia característica da derivada direcional, no entanto as maiores perdas relativas ocorreram em baixas frequências (até $20 \mathrm{~Hz}$ ), devido á atenuação do ground-roll no dado e as altas frequências possuem uma menor perda relativa. É possível notar que, apesar de evidenciar os conteúdos de altas frequência, o método não elimina completamente as informações de baixa frequência, assim, informações de interesse de baixa frequência ainda podem ser observadas no dado.

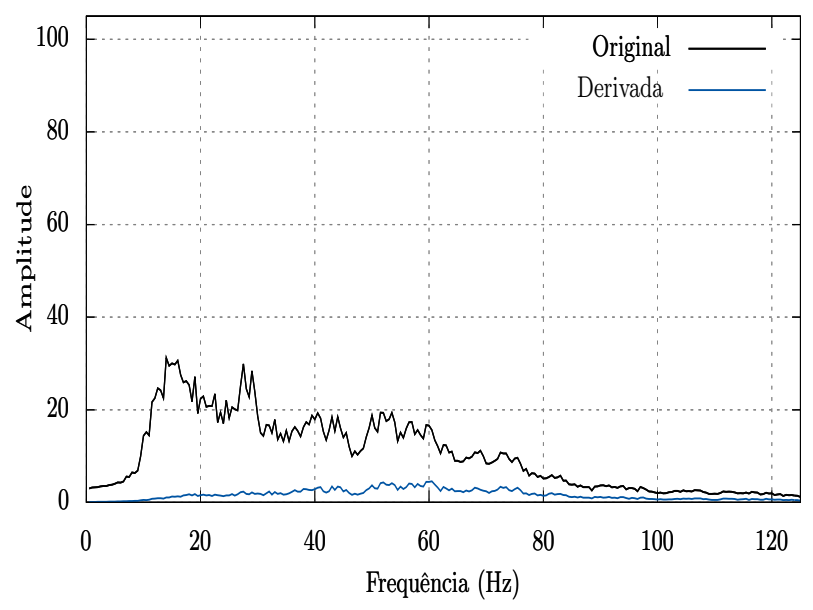

Figura 3: Espectro de amplitude média do tiro 39

A Figura 4a mostra o espectro fk antes da aplicação da filtragem e a Figura $4 b$ após a aplicação do filtro no tiro 39. É possível notar que houve uma maior concentração 
de energia na área referente a reflexão na Figura $4 b$, havendo uma atenuação nas energias da onda direta e ground-roll no sismograma. Assim como o espectro de potência, o espectro fk demonstra que eventos de baixas frequência não são perdidos e eventos de alta frequência são ressaltados.

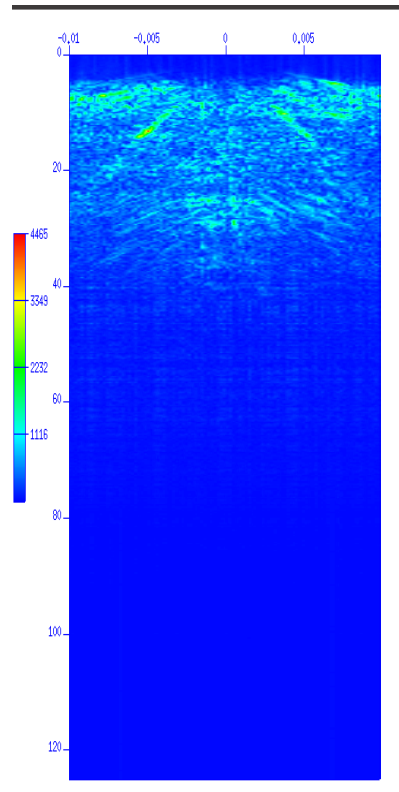

(a)

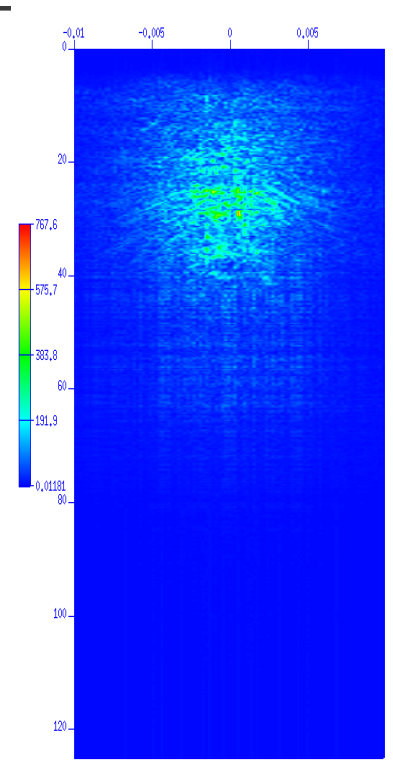

(b)
Figura 4: (a) Espectro fk original (b) espectro fk após a filtragem.

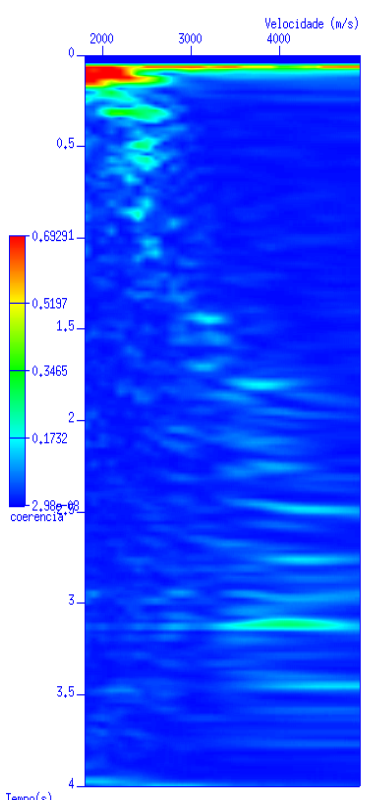

(a)

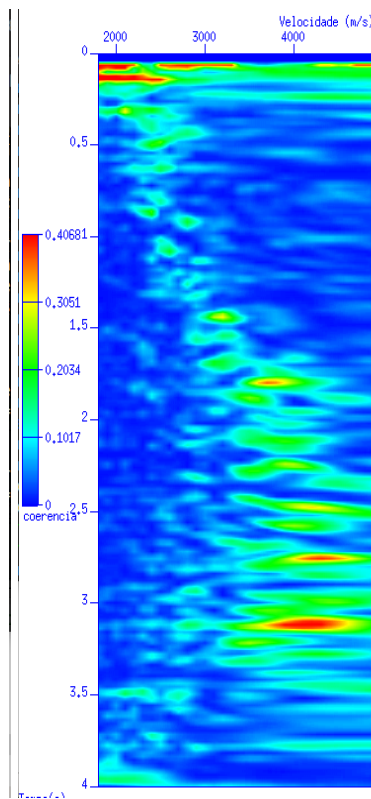

(b)
Figura 5: (a)Espectro de velocidade do tiro 39 original (b) Espectro de velocidade d tiro 39 filtrado

A Figura 5 a mostra o espectro de velocidade do tiro 39 antes da filtragem, enquanto a Figura $5 \mathrm{~b}$ mostra o espectro de velocidade após a filtragem. É possível notar que houve um forte aumento na coerência, tornando evidente 0 incremento da razão sinal/ruído após a filtragem, no entanto é possível notar que ainda há uma energia esparramada nos segundos iniciais do espectro de velocidades. Isto ocorre principalmente devido a onda direta, pois apesar do método atenuar este evento ele não é tão atenuado quanto o ground-roll.

A Figura 6 mostra a linha L5090 empilhada sem a filtragem enquanto a Figura 7 mostra a mesma seção empilhada após a realização da filtragem, ambas as seções foram empilhadas com o mesmo campo de velocidades, que foi gerado a partir da análise de velocidades do dado filtrado, desta forma a única diferença entre as seções é a aplicação do filtro. É possível notar que a seção não filtrada contém diversos eventos lineares que pouco aparecem na seção filtrada, este ganho na razão sinal/ruído é mais evidente nas reflexões mais profundas, onde os eventos lineares de alta amplitude foram mais atenuados quando comparados ao dado original.

\section{Conclusões}

A derivada radial direcional é um poderoso método para atenuação de ground-roll, neste trabalho foi proposto uma forma de construção do filtro utilizando a derivada direcional analítica da função peso do método de Shepard. Através de diferentes análises do tiro 39 e da linha L5090 como um todo, bem como, a análise da seção empilhada. Foi demonstrado que o filtro funciona, ressaltando conteúdos de alta frequência e atenuando conteúdos lineares de baixa frequência, no entanto, sem descartar completamente as informações de baixa frequência, assim sendo possível que mesmo após esta filtragem eventos de baixa frequência sejam observados no sismograma. O método, no entanto, não se mostrou completamente eficaz na atenuação da onda direta, sendo necessário que esta seja melhor atenuada nas etapas seguintes do processamento.

\section{Agradecimentos}

Nós agradecemos FINEP, FAPESB, PETROBRAS, ANP e CNPq (Projeto INCT-GP), Brasil por suporte financeiro e a Landmark pelas licenças educacionais concedida ao centro de pesquisa em Geofísica e Geologia (CPGGUFBA).

\section{Referências}

Manenti, R., 2013, O uso da transformada radial e filtros svd e derivada temporal $2 \mathrm{~d}$ para atenuação do groundroll: Trabalho de Conclusão de curso. Universidade Federal da Bahia, Salvador, Brasil.

Ottolini, R., 1979, Migration of radial trace sections: Standford Exploration Project Report, SEP-20, 97-115.

Santos, S., 2014, Atenuação do ground-roll utilizando a filtragem directional radial: Trabalho de Conclusão de curso. Universidade Federal da Bahia, Salvador, Brasil.

Shepard, D., 1968, A two-dimensional interpolation function for irregularly-spaced data: Proceedings of the 1968 23rd ACM National Conference, 517-524, ACM. 


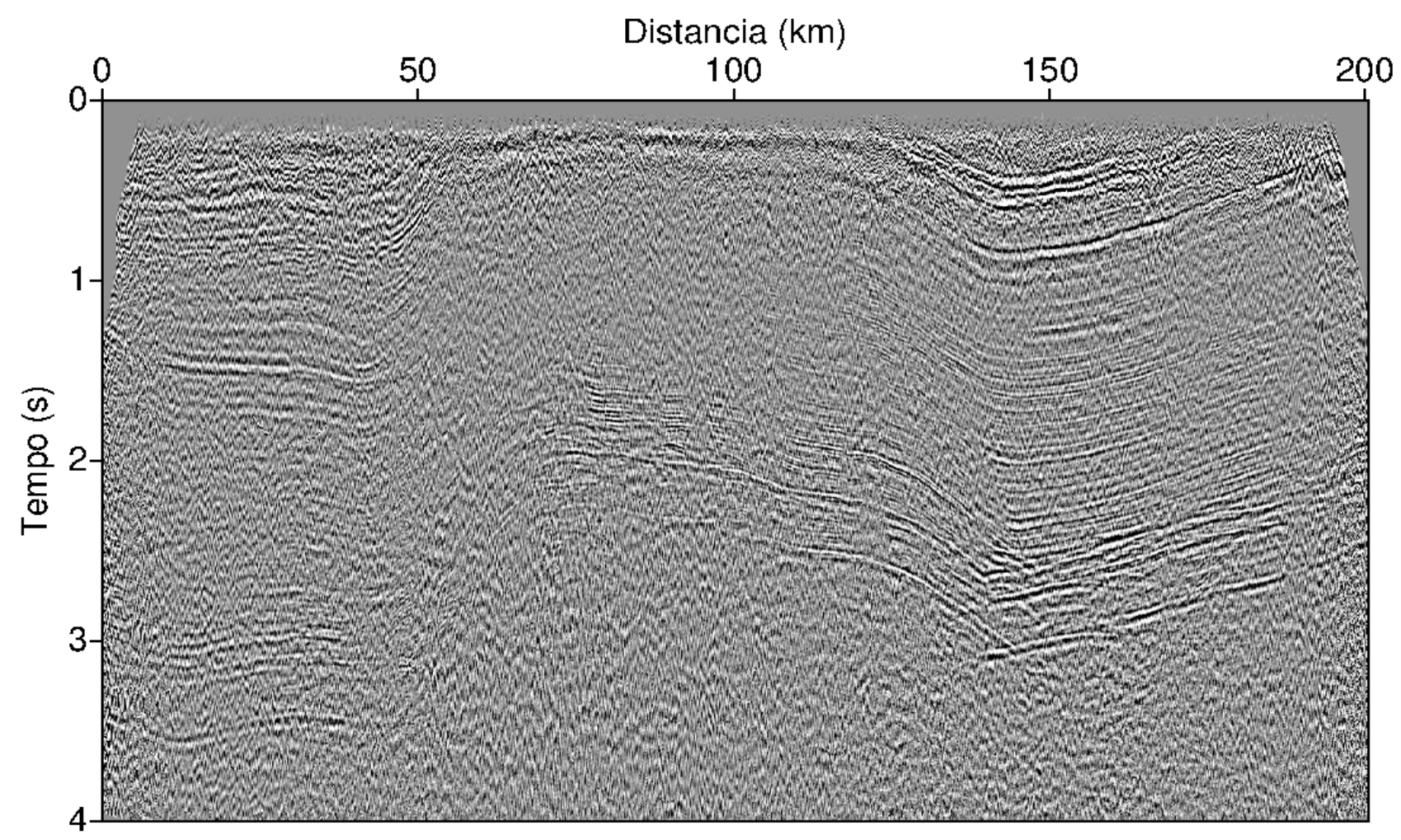

Figura 6: Seção empilhada original.

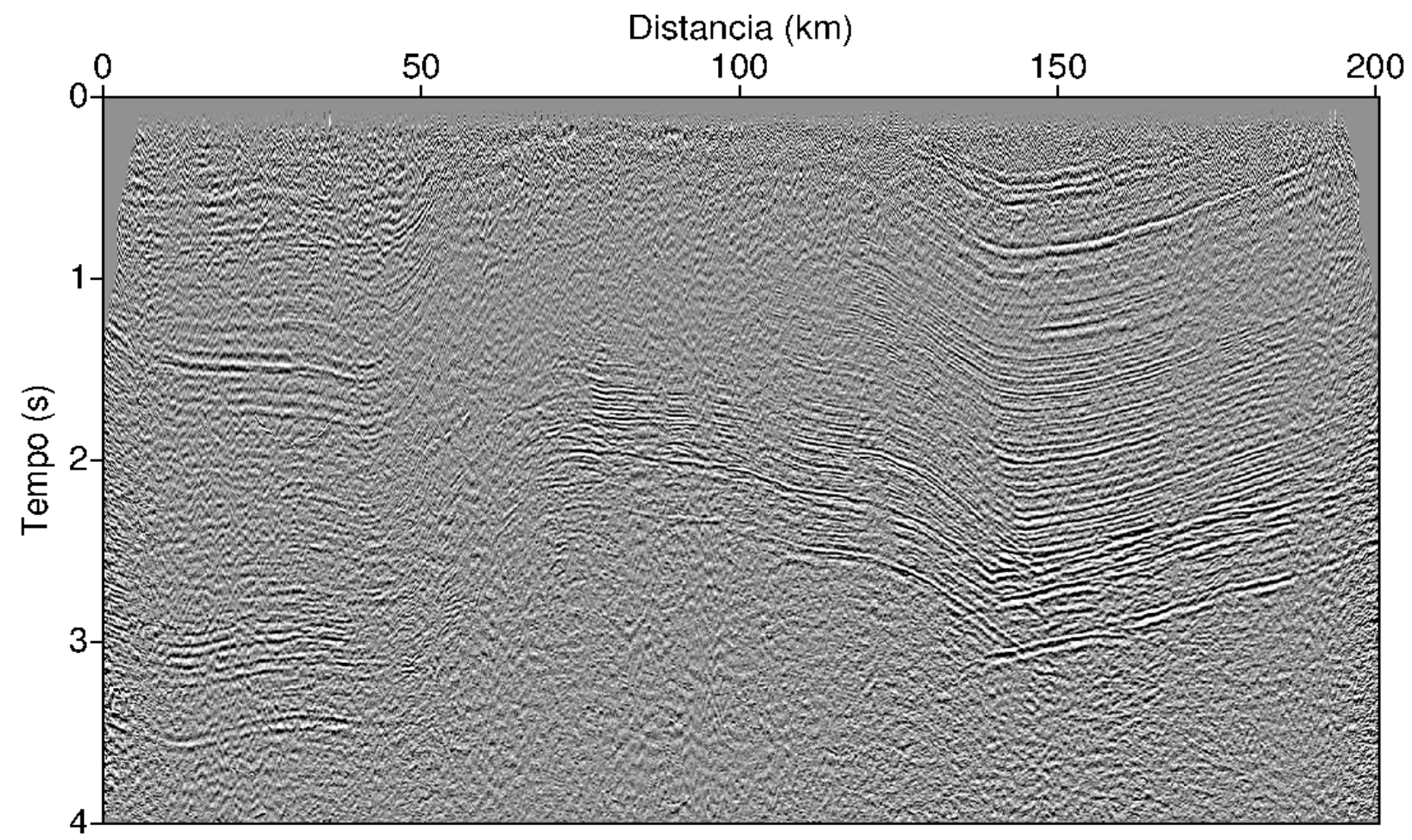

Figura 7: Seção empilhada após filtragem. 